\title{
Tecnura
}

INVESTIGACIÓN

\section{Modelación y simulación computacional del proceso de evaporación osmótica}

\section{Modeling and computational simulation of the osmotic evaporation process}

\author{
Freddy Forero Longas ${ }^{1}$, Adriana Patricia Pulido Díaz², Sergio Andrés Cabrera Navarro ${ }^{3}$
}

Cómo citar: Forero Longas, F., Pulido Diaz, A. P., \& Cabrera Navarro, S. A. (2016). Modelación y simulación computacional del proceso de evaporación osmótica. Revista Tecnura, 20(49), 29-44. doi: 10.14483/udistrital.jour. tecnura.2016.3.a02

Fecha de recepción: 10 de octubre de 2014

\section{RESUMEN}

Contexto: Dentro de las tecnologías de procesamiento con membranas, la evaporación osmótica es una alternativa promisoria para la transformación de frutas exóticas, generando productos concentrados que pueden ser usados en la alimentación diaria, siendo más fáciles de consumir, disminuyendo gastos de transporte e incrementando la vida útil.

Método: En este trabajo de investigación se estudió y desarrollo una estrategia integral para la modelación y simulación multifísica de los fenómenos de transferencia de masa y movimiento en el proceso de evaporación osmótica, por medio del software Comsol $^{\circledR}$ y Matlab $^{\circledR}$, usando un enfoque de geometría axial en dos dimensiones como simplificación del módulo real y el método de elementos finitos para la solución numérica, las simulaciones fueron validadas experimentalmente en un sistema de evaporación osmótica a escala de laboratorio.

Resultados: Los modelos utilizados y las simulaciones generadas fueron estadísticamente significativas $(p<0,05)$ en predecir el comportamiento del flux, teniendo en cuenta el efecto de la velocidad y temperatura de alimentación, junto con la
Fecha de aceptación: 15 de febrero de 2016

velocidad de la salmuera, obteniéndose correlaciones mayores al 96\% entre los datos experimentales y los calculados.

Conclusiones: Se encontró que para las condiciones estudiadas el modelo difusional Knudsen es el más adecuado en describir la transferencia del vapor de agua a través de la membrana hidrófoba; las simulaciones desarrolladas describen de forma adecuada el proceso de evaporación osmótica, convirtiéndose en una herramienta para el desarrollo más rápido y económico de esta tecnología.

Palabras clave: difusión, membrana hidrófoba, transferencia de calor, transferencia de masa.

\section{ABSTRACT}

Context: Within the processing technologies with membranes, osmotic evaporation is a promising alternative for the transformation of exotic fruits, generating concentrated products that can be used in the daily diet, being easier to consume, reducing transportation costs and increasing shelf life.

Method: In this research, it was studied and developed a comprehensive strategy for multiphysics modeling and simulation of mass and momentum

Ingeniero Agroindustrial, doctor en Ingeniería de Alimentos, profesor asistente de la Universidad de Antioquia. Medellín, Colombia. Contacto: freddy.forero@udea.edu.co

2 Ingeniera Agroindustrial, estudiante Doctorado en Ingeniería, Universidad del Valle. Cali, Colombia. Contacto: adriana.pulido@correounivalle.edu.co

Ingeniero Agroindustrial, coordinador Red Tecnoparque Nodo La Granja-Sena Regional Tolima. Tolima, Colombia. Contacto: sacabrera40@ misena.edu.co 
transfer phenomena in the process of osmotic evaporation through Comsol ${ }^{\circledR}$ and Matlab $₫$ software. It was used an axial geometry approach in two dimensions as simplifications of real module and the finite element method for the numerical solution. The simulations were validated experimentally in an osmotic evaporation system of laboratory scale.

Results: The models used and the generated simulations were statistically significant $(p<0,05)$ in predicting the flux behavior, taking into account the effect of flow and temperature feed together with the brine flow, being obtained correlations above $96 \%$ between experimental and calculated data.

Conclusions: It was found that for the conditions studied the Knudsen diffusion model is most suitable to describe the transfer of water vapor through the hydrophobic membrane. Simulations developed adequately describe the process of osmotic evaporation, becoming a tool for faster economic development of this technology.

Keywords: diffusion, hydrophobic membrane, heat transfer, mass transfer.

\section{INTRODUCCIÓN}

Las tecnologías de membranas tienen ventajas sobre otros procesos de conservación, ya que las características del producto se mantienen mejor, se utilizan temperaturas bajas; además existen requerimientos de energía bajos, reducidas exigencias de espacio y flexibilidad para operar. Entre esta gama de operaciones con membranas, la concentración por evaporación osmótica $(\mathrm{EO})$ tiene potencial para ser usada en las industrias cosmética, alimentaria, farmacéutica y química; igualmente, esta técnica ha generado mucho interés en el campo de los concentrados de jugos de frutas, vegetales, café instantáneo, leche, té y otros materiales sensibles al calor (Shaw et al., 2001; Vaillant et al., 2012; Valdés et al., 2013), debido a que se realiza a temperatura ambiente, presión atmosférica y condiciones isotérmicas (Bailey et al., 2012; Nii, Jebson y Cussler, 2002) disminuyendo el pardeamiento no enzimático, pérdida de aromas, degradación de color y sabor.

En la concentración osmótica, el transporte de materia (figura 1A) se divide en tres fases: las etapas inicial y final son el movimiento de agua, desde el producto diluido a través de la interface y desde la zona de condensación, hacia la solución concentrada; la etapa intermedia es cuando el vapor pasa a través de la membrana (Romero, 2003; Thanedgunbaworn, Jiraratananon y Nguyen, 2009). El diferencial de presión en la membrana se produce con soluciones salinas como el $\mathrm{MgSO}_{4}$ $\mathrm{MgCl}_{2}, \mathrm{CaCl}_{2}, \mathrm{NaCl}$, y líquidos como el glicerol, que tienen alta solubilidad, baja actividad de agua y alta tensión superficial. El sistema básico para
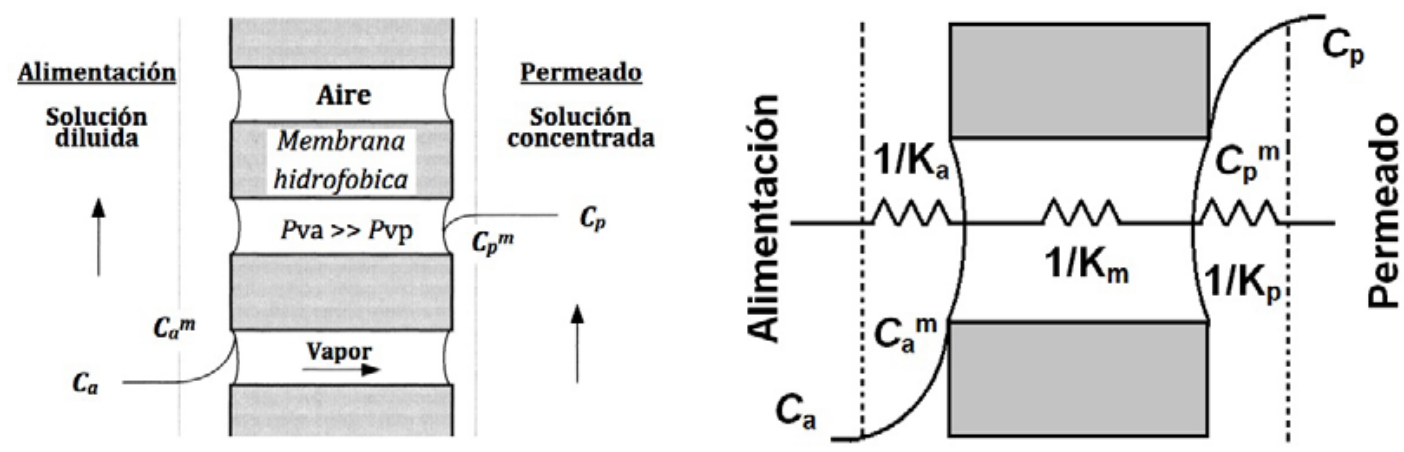

Figura 1. A) Principio del proceso. B) Perfil de concentraciones y resistencia a la transferencia de materia en la EO Fuente: elaboración propia. 
describir la transferencia de masa está dado en la ecuación (1) donde se relaciona el flux $(N)$ y el diferencial de presiones, por medio de una constante $(K)$ que es la permeabilidad de la membrana (Alves y Coelhoso, 2004; Cassano et al., 2003).

$$
\begin{aligned}
\mathrm{HHV} & =0.312 \mathrm{FC}+0.1534 \mathrm{VM} \\
K & =\left(\frac{1}{K_{a}}+\frac{1}{K_{m}}+\frac{1}{K_{p}}\right)^{-1}
\end{aligned}
$$

Como las condiciones existentes en la interface no siempre están disponibles, una representación más completa del proceso está dada por la ecuación, donde el coeficiente global $K$ integra varias resistencias a la transferencia de masa (figura 1B).

Puesto que en los poros de la membrana existe aire, solo dos formas de difusión participan en la transferencia de agua, difusión Knudsen y molecular, según la teoría cinética de los gases (Thanedgunbaworn, Jiraratananon y Nguyen, 2007a). El número de Knudsen $(K n)$ definido por la ecuación se usa para estimar cuál de los mecanismos de difusión es el predominante, comparando la trayectoria media libre $(\lambda)$ de la molécula (ecuación 4) que se difunde con el radio de los poros en la membrana (Qtaishat, Matsuura, Kruczek y Khayet, 2008).

$$
\begin{gathered}
K n=\frac{\lambda}{2 r} \\
\lambda=\frac{k_{b} T}{P \sqrt{2 \pi \sigma^{2}}}
\end{gathered}
$$

Para un tamaño de poro relativamente pequeño, $K n \geq 10$, las moléculas tienden a colisionar frecuentemente con las paredes del poro y el modelo Knudsen representado por las ecuaciones y (6) es el más conveniente.

$$
\begin{aligned}
& N=K_{m}^{k}\left(P_{w a} " P_{w p}\right) \\
& K_{m}^{k}=\frac{1}{3} \frac{\varepsilon d_{p}}{\chi \delta} \sqrt{\frac{8 M}{\pi R T}}
\end{aligned}
$$

Cuando los poros son grandes, $K n \leq 0,01$, las moléculas de gas colisionan más frecuentemente entre sí, la difusión molecular es considerada predominante (Celere y Gostoli, 2002) y el flujo de vapor puede ser descrito por las ecuaciones, (8) y (9) donde $P_{\text {Alm }}$ es la presión media logarítmica dentro de los poros y D (coeficiente difusional) es función de la temperatura y la presión.

$$
\begin{gathered}
N=K_{m}^{M} \frac{P_{w a}-P_{w p}}{P_{A / m}} \\
K_{m}^{M}=\frac{\varepsilon M}{\chi \delta} \frac{P D}{R T} \\
\frac{P D}{T^{b}}=\text { const }
\end{gathered}
$$

Por último, en la región de transición, 0,01< $K n<10$, los dos fenómenos se combinan y en este caso la mejor opción es utilizar un modelo mixto como el de la ecuación (10) con una permeabilidad del tipo $\mathrm{K}_{\mathrm{m}}^{\mathrm{kM}}$ planteada por la ecuación (11), en la cual se incluye el término $P_{\text {Alm }}$ que tiene en cuenta el efecto del aire presente en los poros.

$$
\begin{aligned}
K_{m}^{k M}= & {\left[3 \frac{\chi \delta}{\varepsilon d_{p}} \sqrt{\frac{\pi R T}{8 M}+\frac{\chi \delta}{\varepsilon M}} \frac{R T}{P D}\right]^{-1} } \\
& \frac{1}{K_{m}^{k M}}=\frac{1}{K_{M}}+\frac{1}{P_{A l m} K_{k}}
\end{aligned}
$$

El flux de vapor en las zonas de polarización del lado de la alimentación y del permeado se expresa por las ecuaciones y; algunas investigaciones desprecian esta polarización cuando la alimentación es solo agua, y en condiciones donde se cuantifican otros parámetros de la membrana (Courel et al., 2000; Courel et al., 2001).

$$
\begin{aligned}
& N=k_{a}\left(C_{a}-C_{a}^{m}\right) \\
& N=k_{p}\left(C_{p}-C_{p}^{m}\right)
\end{aligned}
$$

Al tener en cuenta la resistencia por polarización, las concentraciones en las capas límites son calculadas por el modelo presentado en las ecuaciones $y$, donde no se tiene en cuenta el cambio de la distribución de la capa en el módulo.

$$
\begin{gathered}
C_{a}^{m}=C_{a} \exp \left(\frac{N}{\rho_{w} k_{a}}\right) \\
C_{p}^{m}=C_{p} \exp \left(\frac{-N}{\rho_{w} k_{p}}\right)
\end{gathered}
$$


En módulos de fibras huecas, se aplican modelos semiempíricos para estimar el coeficiente $k_{a^{\prime}}$ con lo cual el flujo de alimentación al interior de la fibra se asimila al de una tubería, con la ecuación de Sieder-Tate y las respectivas para transferencia de materia, ecuaciones y (Martínez-Díez, Florido-Díaz y Vázquez-González, 2000).

$$
\begin{gathered}
\text { Si } \quad G z=\operatorname{Re} \cdot \operatorname{Pr} \cdot \frac{d_{h}}{L} \geq 100 \\
S h=\frac{k_{a} d_{h}}{D_{w}}=1,86\left(\mathrm{Re} \cdot S c \cdot \frac{d_{h}}{L}\right)^{1 / 3} \\
G z=\operatorname{Re} \cdot \operatorname{Pr} \cdot \frac{d_{h}}{L}<100 \\
G z m=\operatorname{Re} \cdot S c \cdot \frac{d_{h}}{L} \\
S h=\frac{k_{a} d_{h}}{D_{w}}=3,66+\frac{0,085 \mathrm{Gzm}}{1+0,047 \mathrm{Gzm}^{2 / 3}}
\end{gathered}
$$

Varios estudios han generado modelos para estimar el coeficiente $\left(k_{\mathrm{p}}\right)$ al exterior de las fibras (carcasa) (Gawronski y Wrzesinska, 2000; Lipnizki y Field, 2001; Wu y Chen, 2000). Recientemente (Thanedgunbaworn, Jiraratananon y Nguyen, 2007b) se ha desarrollado una nueva ecuación, con la cual se logra describir mejor el fenómeno, hallando que el número de Reynolds es función del empaquetamiento $(\varnothing)$, tal como se presenta en la ecuación (19).

$$
\begin{gathered}
S h=\left(-0,45 \varnothing^{2}+0,39 \varnothing-0,04\right) \cdot \operatorname{Re}^{\left(4,01 \varnothing^{2}-4,42 \varnothing+1,55\right)} S c^{0,33} \\
\varnothing=\# \text { Fibras } \times\left(\frac{d_{o}}{d_{i}}\right)^{2}
\end{gathered}
$$

\section{METODOLOGÍA}

El módulo de fibras huecas (MFH) constituye la parte principal del sistema a modelar, cada fibra se compone de un cilindro con radio $(r)$ y la longitud (z) rodeado por una membrana porosa que actúa de forma independiente, la alimentación (agua) fluye con un comportamiento laminar por dentro de la fibra (lumen), siendo introducido a $z=0$, mientras que la solución de extracción (salmuera) es pasada a través de la carcasa del módulo, provocando la remoción de agua en forma de vapor desde la alimentación y que por subsecuente difusión a través de la membrana es absorbida por la salmuera. La geometría del módulo fue simplificada como se muestra en la figura 2, con el fin de facilitar el análisis y reducir el consumo de recursos computacionales, por lo que se utilizó un enfoque de simetría axial, donde se aprecian tres subdominios lumen, membrana y carcaza, que pueden ser identificados también como agua, membrana y salmuera.
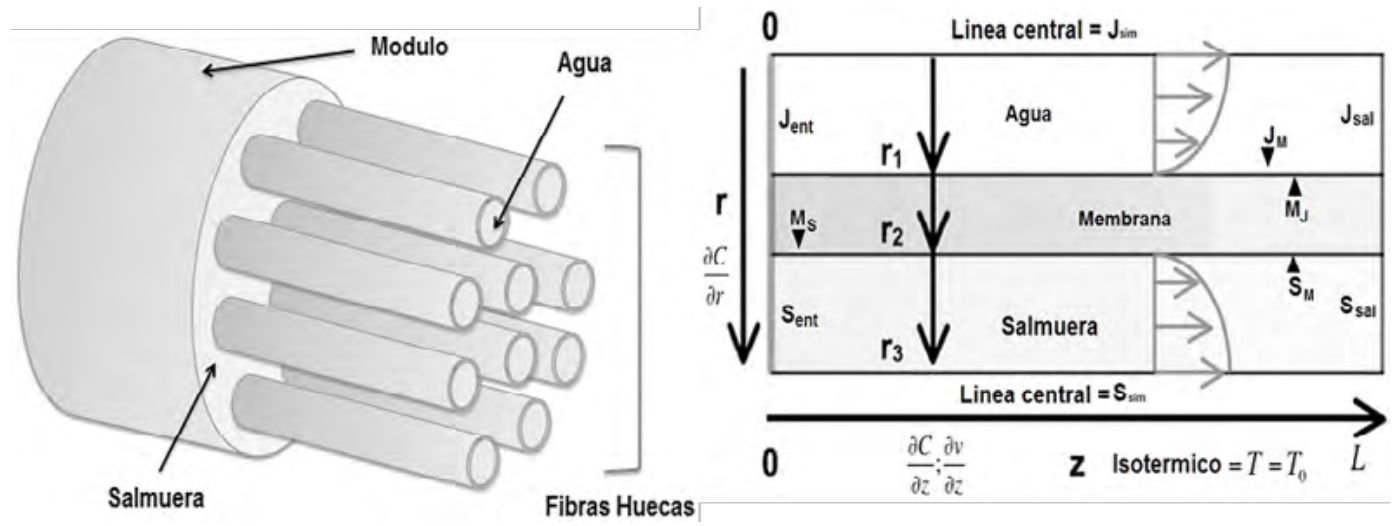

Figura 2. Geometría general del módulo de fibras huecas (izq.), dominios y límites para la simetría de la fibra (der) Fuente: elaboración propia. 
El modelo de la superficie libre de Happel (Chen, Gao, Jin y Zheng, 2011) fue usado para estimar el radio $r_{3}$ en el lado de la salmuera; de acuerdo con este enfoque, se considera que cada fibra en el espacio de la carcasa está rodeada por un fluido envolvente, en el cual no existe transferencia de momento, masa o calor en la superficie externa. Las fibras se consideran homogéneamente distribuidas y el flujo es puramente axial; el modulo es considerado como una serie de celdas de superficies libres, cada celda tiene solo una fibra en el centro y está rodeada de fluido uniforme, matemáticamente la superficie libre es definida por las ecuaciones (20) y (21).

$$
\begin{gathered}
r_{3}=\left(\frac{1}{1-\phi}\right)^{1 / 2} r_{2} \\
1-\phi=\frac{n r_{2}^{2}}{r_{i m}{ }^{2}}
\end{gathered}
$$

Donde $\Phi$ es la fracción volumétrica de vacío y puede ser calculada usando la ecuación, para cual $n$ es el número de fibras y $r_{i m}$ es el radio interior del módulo.

\section{Ecuaciones gobernantes}

La ecuación (22) representa el estado estacionario para el transporte de vapor de agua en el lado del lumen $\left(C_{1}\right)$ y puede ser escrita como:

$v_{z} \frac{\partial C_{1}}{\partial z}=D_{w}\left(\frac{\partial^{2} C_{1}}{\partial r^{2}}+\frac{1}{r} \frac{\partial C_{1}}{\partial r}+\frac{\partial^{2} C_{1}}{\partial z^{2}}\right)$

Donde $D w$ es el coeficiente de auto difusión del agua (Holz, Heil y Sacco, 2000) y es descrito por la ecuación (23):

$$
D_{w}=\left(1,635 \times 10^{-8}\right)\left(\frac{T}{215,05}-1\right)^{2.063}
$$

La ecuación es la expresión para el transporte de agua $\left(C_{2}\right)$ al interior de la membrana, el cual es considerado debido solamente a la difusión.

$$
D_{w a}\left(\frac{\partial^{2} C_{2}}{\partial r^{2}}+\frac{1}{r} \frac{\partial C_{2}}{\partial r}+\frac{\partial^{2} C_{2}}{\partial z^{2}}\right)=0
$$

En los poros de la membrana existe aire que viene mezclado con la alimentación y solo la difusión Knudsen $\left(D_{k}\right)$ y molecular $\left(D_{m}\right)$ están involucradas en la transferencia de vapor; para tener en cuenta estas dos contribuciones y obtener el valor de $D_{\text {wa }}$ que representa el coeficiente de difusión efectivo del vapor de agua en el aire retenido dentro de los poros, se hizo uso de la aproximación de Bonsaquet representada por las ecuaciones (26) y (27) (Rezakazemi, Shirazian y Ashrafizadeh, 2012):

$$
\frac{1}{D_{w a}}=\frac{1}{D_{k}}+\frac{1}{D_{m}}
$$

Donde $D_{k}=\left(\frac{\varepsilon d_{p}}{3 \tau}\right)\left(\frac{8 R T}{\pi M_{w}}\right)^{0.5}$

$$
D_{m}=\left(\frac{\varepsilon}{\tau}\right)\left(-2,775 \times 10^{-6}+4,479 \times 10^{-8} T+1,656 \times 10^{-10} T^{2}\right)
$$

En las anteriores ecuaciones, $\varepsilon, \tau, d_{p}$ son la tortuosidad, porosidad y diámetro de poro en la membrana; $M_{w^{\prime}}$ la masa molar del agua; $R$, constante de los gases; $T$, temperatura absoluta. La ecuación (28) calcula la transferencia del vapor de agua en estado estacionario para el lado de la carcasa $\left(C_{3}\right)$ del contactor en coordenadas cilíndricas, se obtiene a través de la ley de difusión de Fick:

$$
v_{z} \frac{\partial C_{3}}{\partial z}=D_{w s}\left(\frac{\partial^{2} C_{3}}{\partial r^{2}}+\frac{1}{r} \frac{\partial C_{3}}{\partial r}+\frac{\partial^{2} C_{3}}{\partial z^{2}}\right)
$$

La difusión del agua en soluciones salinas puede ser vista como la auto difusión del agua retardada por la presencia de los iones en solución, el coeficiente de autodifusión del agua presenta un máximo valor en el límite de la dilución extrema y este tiende a cero en el límite de la sal anhidra (Suárez-Iglesias, Medina, Pizarro y Bueno, 2008). El coeficiente de difusión del agua en la salmuera $\left(D_{w s}\right)$ puede ser calculado con la ecuación, donde $D_{w}$ es determinado con la expresión y $x_{s}$ es la fracción de sal en la solución.

$$
D_{w s}=D_{w}\left(1-\left(1+\left(\frac{\sqrt{x_{b}}}{0,55}\right)^{-5,52}\right)^{-0,56}\right)
$$




\section{Condiciones límite}

A la entrada de una fibra, se asume que el flujo está completamente desarrollado bajo un régimen laminar y se especifica un flujo parabólico según la ecuación (30), y a la salida de la fibra, la presión es la atmosférica, según se especifica en la ecuación (31).

$$
\begin{gathered}
z=0 \rightarrow V_{z}=V_{a l m}\left(1-\left(\frac{r}{r_{1}}\right)^{2}\right) \\
z=L \rightarrow P=P_{a t m}
\end{gathered}
$$

La línea central interna de la fibra es una simetría axial, definida por la ecuación (32).

$$
r=0 \rightarrow \frac{\partial v_{z}}{\partial r}=0 \rightarrow v_{r}=0
$$

En la superficie de la membrana, la velocidad axial $\left(v_{z}\right)$ es establecida como cero $(0)$ o condición de no deslizamiento, especificada por la ecuación (33). A la entrada de una fibra, la concentración inicial de agua en la alimentación (34) es $C_{10}$, la cual corresponde a la inicial.

$$
\begin{gathered}
r=r_{1} \rightarrow v_{z}=0 \\
z=0 \rightarrow C_{1}=C_{J 0}
\end{gathered}
$$

La condición de frontera para el flujo convectivo asume que la masa que pasa por la frontera $z$ $=\mathrm{L}$ es dominada por la convección. En otras palabras, se supone que el flujo de masa debido a la difusión a través de este límite es cero (35), la línea central de la fibra es una simetría axial (36):

$$
\begin{gathered}
z=L \rightarrow-D \frac{\partial C_{1}}{\partial z}=0 \\
r=0 \rightarrow \frac{\partial C_{1}}{\partial z}=0 \\
z=0 \rightarrow v_{z}=\left(\frac{Q_{S}}{\left(\pi \cdot r_{m}^{2}\right)-\left(n \cdot \pi\left(r_{1}+r_{2}\right)^{2}\right)}\right)\left(1-\left(\frac{r}{r_{3}}\right)^{2}\right) \times \frac{\left(r / r_{3}\right)^{2}-\left(r_{2} / r_{3}\right)^{2}+2 \ln \left(r_{2} / r\right)}{3+\left(r_{2} / r_{3}\right)^{4}-4\left(r_{2} / r_{3}\right)^{2}+4 \ln \left(r_{2} / r_{3}\right)} \\
\text { huecas no está absolutamente } \\
\text { modelo de Happel, este se } \\
\text { Huang, } \text { Chi y Pei, 2012). }
\end{gathered}
$$

En la superficie de la membrana para la concentración $\left(\mathrm{C}_{1}\right)$, se establece la siguiente condición especial de flux (37), que es descrita más en detaIle en las condiciones límite de la membrana.

$$
r=J_{M} \rightarrow-D \frac{\partial C_{1}}{\partial z}=\mathrm{M}\left(\mathrm{C}_{2}-K * \mathrm{C}_{1}\right)
$$

Debido a que existen discontinuidades del perfil de concentración en los límites entre los líquidos y la membrana, se deben utilizar tres variables separadas para describir la concentración en las respectivas fases; para obtener un flux continuo a través de los límites, se aplica una condición de frontera especial Ilamada el método stiff-spring, en lugar de definir las condiciones de concentración tipo Dirichlet de acuerdo con el coeficiente de partición (K), lo que destruiría la continuidad del flujo. Por medio de las ecuaciones (38) y (39) podemos definir condiciones de flux continuo que, al mismo tiempo, fuerzan las concentraciones a los valores deseados:

$$
\begin{gathered}
r=M_{J} \rightarrow C_{2}=C_{1} \exp \left(\frac{N}{\rho_{w} k_{a}}\right) \\
r=M_{S} \rightarrow C_{2}=C_{3} \exp \left(\frac{N}{\rho_{w} k_{p}}\right)
\end{gathered}
$$

Donde los coeficientes $k_{a}$ y $k_{p}$ se calculan a partir de las ecuaciones y. Por último, con la ecuación (40) se establece que no hay transferencia de masa en ambos bordes de la membrana:

$$
z=0 \rightarrow z=L \rightarrow-D \frac{\partial C_{2}}{\partial z}=0
$$

Para el flujo de salmuera sobre la fibra, el modelo de superficie libre de Happel se usa para caracterizar el perfil de velocidad fuera de las fibras (41). Aunque el flujo en el módulo real de fibras huecas no está absolutamente de acuerdo con el modelo de Happel, este se ha utilizado ampliamente para contactores de membrana (Zhang, 
A la salida de la carcasa, la presión es la atmosférica y se especifica por la ecuación (42), la línea central entre las fibras se consideró una simetría axial (43).

$$
\begin{gathered}
z=L \rightarrow P=P_{a t m} \\
r=r_{3} \rightarrow \frac{\partial v_{z}}{\partial r}=0 \rightarrow v_{r}=0
\end{gathered}
$$

En la superficie de la membrana del lado de la salmuera, la velocidad axial $\left(v_{z}\right)$ es establecida como cero y siguiendo la ecuación (44), en la entrada de la carcasa, se toma la concentración de agua $\left(C_{3}\right)$ presente en el tanque de salmuera, convenientemente convertida en unidades de presión de vapor (45).

$$
\begin{gathered}
r=r_{2} \rightarrow v_{z}=0 \\
z=0 \rightarrow C_{3}=C_{s 0}
\end{gathered}
$$

La condición de frontera para el flujo convectivo asume que la masa que pasa por la frontera $z=$
$L$ es dominada por la convección, se supone que el flujo de masa debido a la difusión a través de este límite es cero (46), la línea central de la fibra es una simetría axial (47).

$$
\begin{gathered}
z=L \rightarrow-D \frac{\partial C_{3}}{\partial z}=0 \\
r=0 \rightarrow \frac{\partial C_{1}}{\partial z}=0
\end{gathered}
$$

En la superficie de la membrana (lado salmuera) para la concentración $\left(\mathrm{C}_{3}\right)$, se establece también la condición especial de flux, que se describió en las condiciones de la membrana (48).

$$
r=S_{M} \rightarrow-D_{w s} \frac{\partial C_{3}}{\partial z}=\mathrm{M}\left(\mathrm{C}_{2}-K * \mathrm{C}_{3}\right)
$$

\begin{tabular}{|c|c|c|c|}
\hline Fluido & Propiedad & Modelo & Fuente \\
\hline \multirow{3}{*}{ Agua } & Presión vapor & $P v=\left(10^{8,07-1730,63 /(233,42+T)}\right) \times 133,32$ & \multirow{3}{*}{$\begin{array}{l}\text { (Wang, Wang, } \\
\text { Duan, An, \& } \\
\text { Lee, 2014) }\end{array}$} \\
\hline & Viscosidad & $\eta=e^{-3,71+\frac{578,91}{-137,54+T}}$ & \\
\hline & Densidad & $\rho_{H_{2} O}(\tau)=\rho_{c, H_{2} O}\left(\begin{array}{l}1+B_{0} \tau^{\frac{1}{3}}+B_{1} \tau^{\frac{2}{3}}+B_{2} \tau^{\frac{5}{3}}+ \\
B_{3} \tau^{\frac{16}{3}}+B_{4} \tau^{\frac{43}{3}}+B_{5} \tau^{\frac{110}{3}}\end{array}\right.$ & \\
\hline \multirow{3}{*}{ Salmuera } & Presión vapor & $\begin{array}{c}P v=\left[\left(10^{8,07-1730,63 /(233,42+T)}\right) \times 133,32\right] \times a_{w} \\
a_{w}=\frac{1-C}{1-0,83766 C} \exp \left[-86,68\left(\frac{C}{6,16-5,16 C}\right)^{2}\right] \times \\
{\left[\left(-9561,7 C^{3}+2853,8 C^{2}-422,4 C\right) \times\left(\frac{1}{T}-\frac{1}{298,15}\right)\right]}\end{array}$ & $\begin{array}{l}\text { (Bui, Nguyen, } \\
\text { \& Joachim, } \\
\text { 2003) }\end{array}$ \\
\hline & Viscosidad & $\mu=0,0007934 \times \exp (0,3719 * \mathrm{C}) \times \exp \left(\frac{2068,075}{T}\right)$ & $\begin{array}{l}\text { (Bui y Nguyen, } \\
\text { 2004) }\end{array}$ \\
\hline & Densidad & $\rho_{s o l}\left(\mathrm{x}_{\mathrm{b}}, T\right)=\rho_{H_{2} O}(T) \sum_{i=0}^{3} \rho_{i}\left(\frac{\mathrm{x}_{\mathrm{b}}}{1-\mathrm{x}_{\mathrm{b}}}\right)^{i}$ & $\begin{array}{l}\text { (Wahab y } \\
\text { Mahiuddin, } \\
\text { 2001) }\end{array}$ \\
\hline
\end{tabular}

Los modelos matemáticos que describen las propiedades físicas de los fluidos involucrados en el proceso de evaporación osmótica se resumen en la tabla 1.

Tabla 1. Propiedades físicas de los fluidos

Fuente: elaboración propia. 


\section{Solución numérica}

Las ecuaciones del modelo se resolvieron numéricamente desde un enfoque multifísico (Mendieta, Montaña y Noguera, 2012; Puin, Noguera y Caicedo, 2015) por medio del software Comsol Multiphysics $4.2 \mathrm{~b}$, conjuntamente con Matlab ${ }^{\circ}$ y la herramienta LiveLink', este software emplea el método de elementos finitos (FEM, por sus siglas en inglés); se usó el solucionador PARDISO, la ejecución y resolución se llevó a cabo en un computador personal Intel i7 (4 GHz-16 GB de RAM). El tiempo de cálculo fue de aproximadamente $20 \mathrm{mi}$ nutos para cada experimento, el número máximo de iteraciones se fijó en 250 y la tolerancia relativa era $1 \mathrm{E}-6$; se empleó un factor de escala de 50 en la dirección $z$, debido a la gran diferencia entre el radio y la longitud de las membranas. Con el propósito de corroborar el funcionamiento del modelo se compararon estadísticamente (Statgraphics
Centurion XVI) los resultados generados por el software contra datos experimentales de evaporación osmótica en los que se usó agua destilada como alimentación, evaluando por triplicado las condiciones de proceso que se presentan en la tabla 2.

Tabla 2. Condiciones de proceso usadas en las simulaciones y validación experimental.

\begin{tabular}{|c|c|c|}
\hline Parámetro & Valores & Unidad \\
\hline Flujo alimentación & $0,250,501,01,50$ & $\mathrm{I} / \mathrm{min}$ \\
\hline Temperatura alimentación & 25303540 & ${ }^{\circ} \mathrm{C}$ \\
\hline Concentración salmuera & 44 & $\% p / v$ \\
\hline Flujo salmuera & 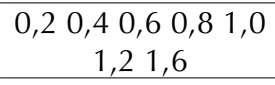 & $\mathrm{I} / \mathrm{min}$ \\
\hline Temperatura salmuera & 25 & ${ }^{\circ} \mathrm{C}$ \\
\hline Modelo difusión & $\begin{array}{l}\text { Knudsen, Fick, } \\
\text { Knudsen +Fick }\end{array}$ & $\mathrm{m}^{2} / \mathrm{s}$ \\
\hline Densidad mallado & $\begin{array}{c}\text { Extragruesa, Grue- } \\
\text { sa, Normal, Fina }\end{array}$ & \# Elementos \\
\hline
\end{tabular}

Fuente: elaboración propia.

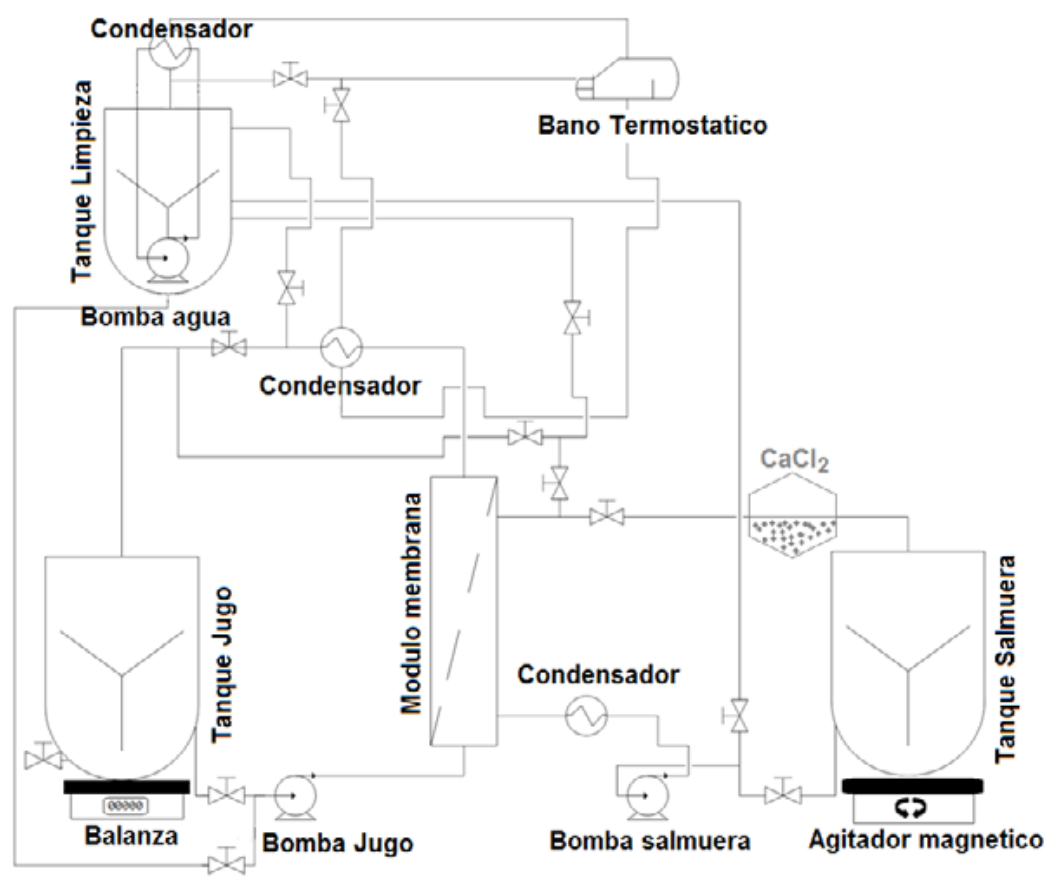

Figura 3. Diagrama del sistema de evaporación osmótica

Fuente: elaboración propia. 


\section{Validación experimental}

El sistema donde se evaporó osmóticamente el agua se presenta en la figura 3, fue utilizado un módulo de fibras huecas que se describe en la tabla 3 , se usaron bombas peristálticas con una velocidad de flujo variable para la solución salmuera y el agua, la salmuera fue mantenida constante a concentración del $44 \%$ p/v de cloruro de calcio $\left(\mathrm{CaCl}_{2}\right)$ por medio de saturación con cloruro nuevo y actividad de agua 0,33 (Aqualab 3TE-Decagon), la relación alimentación/salmuera fue de 1:7 (I), la temperatura del agua se ajustó por medio de un baño termostático, el flux es la pérdida de agua medida cada 10 minutos.

Tabla 3. Características del módulo empleado en la experimentación

\begin{tabular}{lc}
\hline \multicolumn{1}{c}{ Característica } & Módulo \\
\hline Modelo & $\begin{array}{c}\text { Minimodule Liquicel }{ }^{\circledR} \\
1.7 \times 5.5\end{array}$ \\
\hline Material membrana & Polipropileno $\times 50^{\circledR}$ \\
\hline Numero fibras & 7400 \\
\hline Diámetro poro $(\mu \mathrm{m})$ & 0,5 \\
\hline Porosidad $(\%)$ & 40 \\
\hline Longitud fibra $(\mathrm{m})$ & 0,12 \\
\hline Diámetro interno $(\mathrm{um})$ fibra $(\mu \mathrm{m})$ & 220 \\
\hline Diámetro externo $(\mu \mathrm{m})(\mu \mathrm{m})$ & 300 \\
\hline Área de contacto $\left(\mathrm{m}^{2}\right)$ & 0,58 \\
\hline
\end{tabular}

Fuente: elaboración propia.

\section{RESULTADOS}

\section{Modelo difusivo}

Se estableció cuál debería ser el modelo de difusión a utilizar en las posteriores simulaciones, para lo cual se desarrolló un ensayo (triplicado) bajo las siguientes condiciones: flujo "agua" $1 \mathrm{l} / \mathrm{min}$, temperatura $25{ }^{\circ} \mathrm{C},[]_{\text {sal }}: 44 \%$, flujo salmuera 0,2 1,6 l/min, el tamaño de malla fue el sugerido por

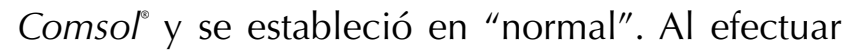
el Anova para comparar los tres modelos utilizados y los datos experimentales se encontraron diferencias significativas $(p<0,05)$, a un nivel del $95 \%$ de confianza. Al realizar la prueba de rangos múltiples (LSD) se encontró que las predicciones generadas por Comsol $^{\circ}$ usando el mecanismo difusional tipo Knudsen, no presentaron diferencias significativas. En la figura 4 se presentan los flux predichos con los modelos contra los valores experimentales; el mejor ajuste, presentado por el modelo Knudsen, se explica por el pequeño tamaño de poro que tiene la membrana $(0,51 \mathrm{um})$, lo cual produce $K n \geq 10$, en donde las moléculas tienden a colisionar frecuentemente con las paredes del poro siendo el modelo Knudsen más conveniente (Liu y Wei, 2014).

Las membranas utilizadas en el proceso de EO no hacen ninguna contribución a la selectividad, su única función es la estabilización de la interfaz

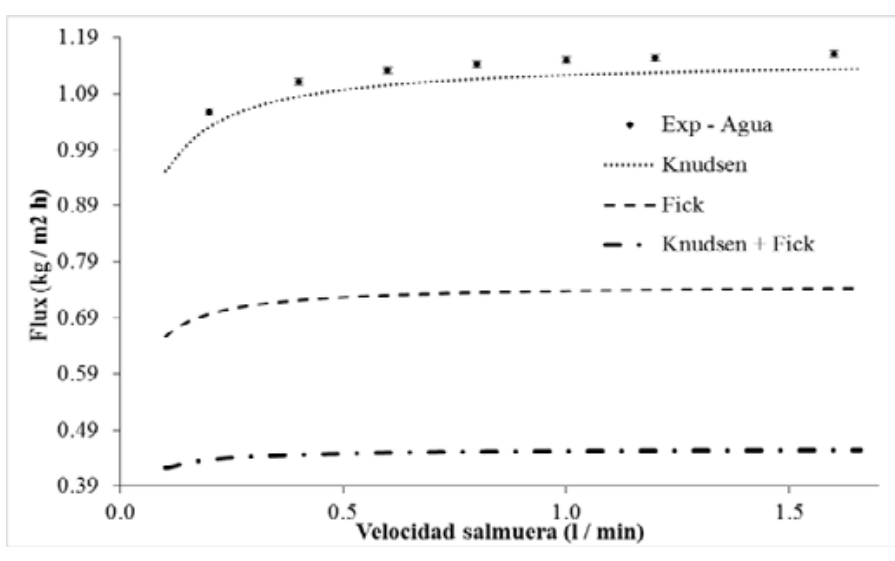

Figura 4. Comparación de los flux predichos por $\mathrm{Comsol}^{p}$ usando diferentes modelos de difusividad

Fuente: elaboración propia. 
entre los dos medios de contacto. Además, la propia membrana está presente como una barrera al flujo de vapor, el efecto de las características de la membrana sobre la transferencia de masa y calor ha sido estudiado por varios investigadores (Domingues, Ramos, Cardoso y Reis, 2014; Viguié et al., 2013; Yang et al., 2013), para tamaños de poro entre 0,05 y 0,2 um, el flux no muestra ser dependiente de este parámetro, en membranas con poros entre 0,2 y 0,45 , se informa de ligeros aumentos en el flujo de vapor, debido principalmente a la dominancia del mecanismos difusional Knudsen. Para membranas con poros mayores a 1 um, se ha observado un incremento significativo en el flux de evaporación a medida que aumenta el tamaño de poro, explicado esto por la marcada influencia de la difusión molecular (Naim, Khulbe, Ismail y Matsuura, 2013; Rahbari-Sisakht, Ismail, Rana y Matsuura, 2013).

\section{Densidad de mallado}

El número de elementos finitos fue el segundo criterio analizado para comprobar los cálculos. La figura 5 presenta la comparación de los flux predichos con el modelo contra los valores experimentales del flux obtenidos para unas condiciones operativas de flujo "agua" $1 \mathrm{l} / \mathrm{min}$, temperatura $25{ }^{\circ} \mathrm{C}$, []$_{\text {sal }}: 44 \%$, flujo salmuera 0,2 - 1,6 l/min y difusión Knudsen, se evidenció que usar un número reducido de elementos, produce resultados de flux bajos, a medida que se refina la malla los resultados se acercan cada vez más a los valores reales.

Tal como lo evidenciaron los resultados del análisis estadístico Anova y la comparación de medias, la mejor correlación se obtuvo al usar una densidad de malla normal, esta no presentó diferencias significativas frente a los datos experimentales, y estaba constituida por 4244 elementos finitos, con lo cual la diferencia entre el flux calculado y el experimental varió entre el 2 y $4 \%$. Con este nivel de mallado, el tiempo de cálculo fue de 5 minutos; seguir aumentando el número de elementos más allá de este límite, no mostró mejoramiento significativo de la calidad de los resultados; por el contrario, ocurrió una ligera sobreestimación de los mismos y se aumentaron los tiempos de convergencia.

\section{Velocidad de alimentación}

Se evaluó estadísticamente cada curva de datos del flux experimental (flujo "agua" 0,25 - 1,5 l/min, temperatura $25{ }^{\circ} \mathrm{C},[]_{\text {sal }}: 44 \%$, flujo salmuera 0,2 - 1,6 l/min y difusión Knudsen) vs. calculados, por medio de una prueba de $t$ para comparar las

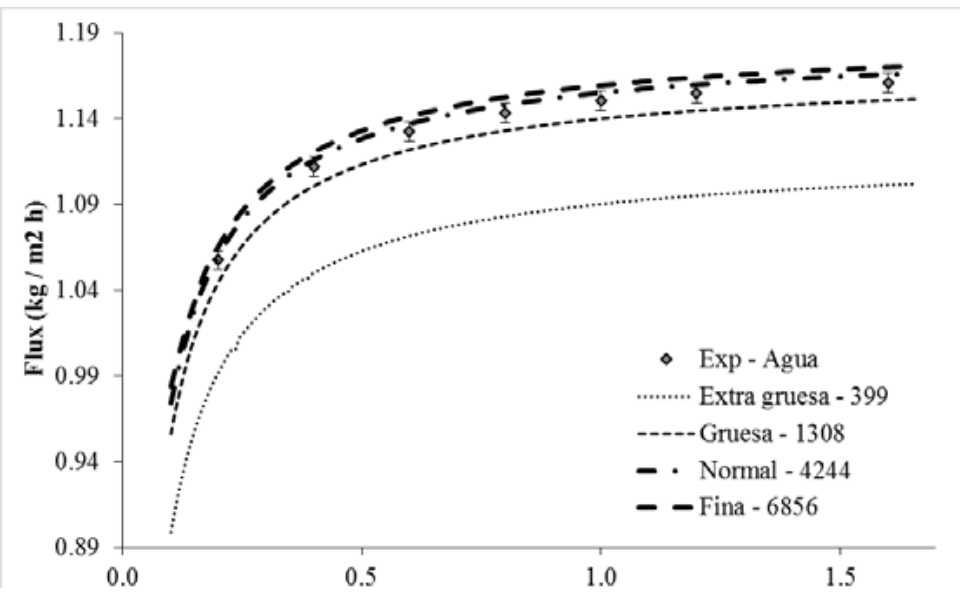

Figura 5. Efecto del tamaño de malla y número de elementos finitos usados para el cálculo del flux

Fuente: elaboración propia. 


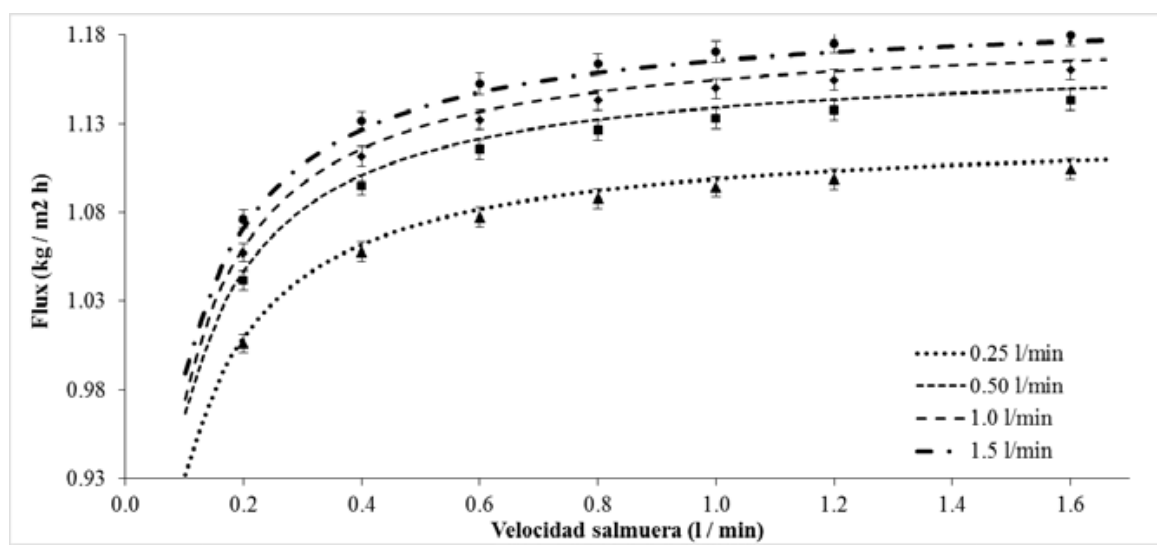

Figura 6. Comparación del flux experimental y los calculados por Comsol' usando diferentes velocidades de alimentación

Fuente: elaboración propia.

medias, a un nivel del $95 \%$ de confianza $(p \leq 0,05)$, teniendo como hipótesis nula: media $_{\text {exp }}=\operatorname{media}_{\text {cal }} y$ la hipótesis alternativa: media exp $_{2}<$ media $_{\text {cal }}$.

Los resultados mostraron que no hubo diferencias significativas entre los datos experimentales y los calculados por Comsol', para ninguna de las 4 velocidades de alimentación; esto confirma la bondad de ajuste del modelo utilizado. Como se puede apreciar en la figura 6, el flux de evaporación aumenta proporcionalmente con la velocidad de la alimentación, este incremento no es indefinido y se limita por las características estructurales y operativas de la membrana (Domingues et al., 2014).

\section{Temperatura de alimentación}

Para el efecto de la temperatura sobre el flux de evaporación, dado en la figura 7, bajo condiciones operativas de: flujo "agua" $1 \mathrm{l} / \mathrm{min}$, temperatura $25-40{ }^{\circ} \mathrm{C}$, $]_{\text {sal }}: 44 \%$, flujo salmuera $0,2-1,6$ I/min y difusión Knudsen; es claro que el flux es exponencialmente dependiente de la temperatura. Este tipo de relación era esperada pues la presión

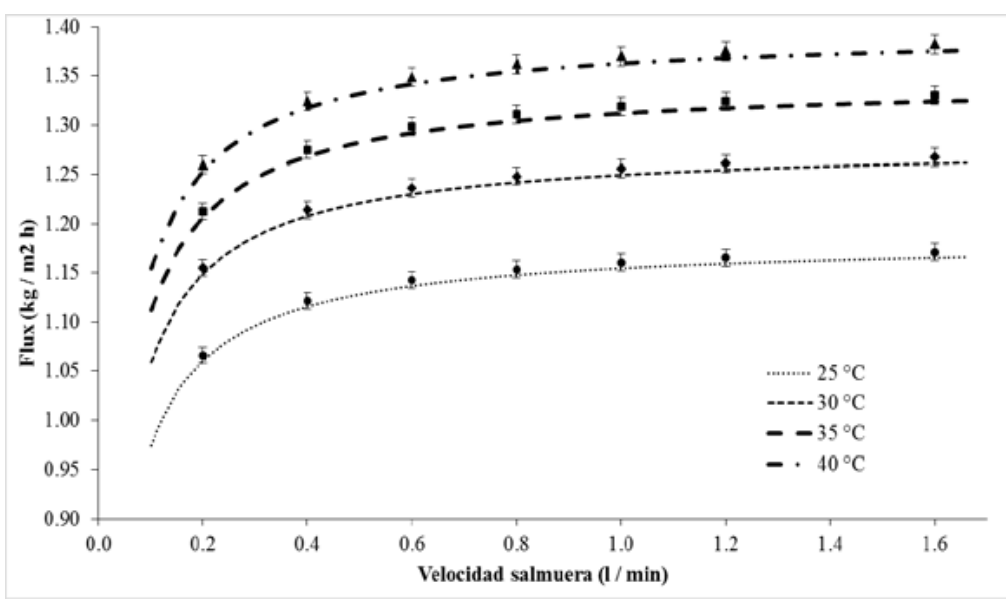

Figura 7. Comparación del flux experimental y los calculados por $\mathrm{Comso}^{\circledR}$ usando diferentes temperaturas de alimentación.

Fuente: elaboración propia. 
de vapor del agua pura presenta este mismo comportamiento (Meng, Ye, Mansouri y Chen, 2014; Zhao et al., 2014).

Matemáticamente, lo anterior puede ser descrito para cualquier par de concentraciones de agua (j) y salmuera (s), con actividades de agua $a_{w j}$ y $a_{w s^{\prime}}$ se tiene que el diferencial de presiones de vapor $\left(P_{v}\right)$ estaría dado por la ecuación (49).

$\Delta P_{v}=\left(a_{w j}-a_{w s}\right) \times P_{0}=\Delta a_{w} \times P_{0}=\Delta a_{w} \times \exp \left(A-\frac{B}{T-C}\right)$

Donde $A, B$ y $C$ son constantes de la ecuación de Antoine, y $T$ es la temperatura de la solución; esta expresión claramente muestra la relación exponencial que se mencionó anteriormente, entre la temperatura y la fuerza impulsora del proceso (Kujawski et al., 2013). Al comparar estadísticamente a un nivel del $95 \%$ de confianza, los resultados experimentales con los predichos para las 4 temperaturas evaluadas, no se encontraron diferencias significativas, los coeficientes de correlación fueron superiores al $97 \%$ en todos los casos; este buen nivel de ajuste por parte del modelo se puede explicar por el hecho de haber utilizado ecuaciones que describen con alto detalle, los fenómenos de transferencia y especialmente las propiedades físicas del agua y la salmuera.

\section{Velocidad de la salmuera}

Este parámetro se mostró consistentemente a través de todas las verificaciones realizadas. Incrementar la velocidad de la salmuera provoca un mayor flux de evaporación. La figura 8 presenta las tres zonas identificadas en el comportamiento del flux: en la zona I este crecimiento es el más rápido, para velocidades entre los 0,1 y $0,4 \mathrm{l} / \mathrm{min}$ con un incremento de $15 \%$ en el flux, la segunda zona comprendida desde 0,4 hasta 1,0 l/min presenta una fase de estabilización llegándose a un máximo de eficiencia cuando el flujo alcanza los 1,0 l/min, pasado este límite se establece un estancamiento o meseta (zona III), donde los incrementos en el flux son muy bajos $(<0,9 \%)$.

La principal explicación de este crecimiento progresivo del flux es el aumento en el coeficiente de transferencia de materia en el lado de la carcasa (Manawi et al., 2014), por el aumento en la turbulencia y también el posible efecto de limpieza sobre la membrana que se ejerce al aumentar la velocidad, retirando depósitos del cloruro de calcio, que pudieran estar acumulados debido a la intrincada geometría formada por el empaquetamiento de las fibras en el módulo; este tipo de colmatación de la membrana no se evidencio durante los experimentos.

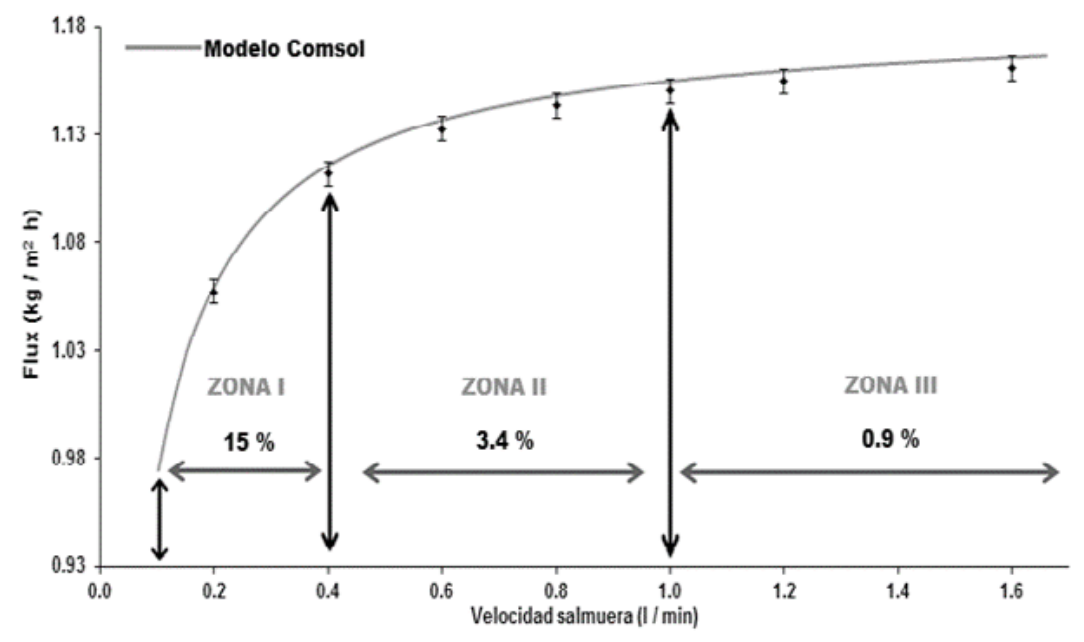

Figura 8. Efecto del flujo de salmuera sobre el comportamiento del flux de evaporación

Fuente: elaboración propia. 


\section{CONCLUSIONES}

El modelo difusional Knudsen fue el más adecuado en describir la transferencia del vapor de agua a través de la membrana, explicado esto por las características estructurales de la membrana que hacen predominar este modelo sobre la difusión molecular; siempre ha de verificarse de forma experimental la selección del modelo difusivo, porque este puede cambiar rápidamente de un tipo a otro con pequeños cambios en el proceso o la membrana. El uso de una geometría axial en dos dimensiones como simplificación del módulo real de evaporación osmótica es una decisión adecuada que permite reducir de forma significativa los recursos de cómputo necesarios para resolver las ecuaciones diferenciales sobre los tres dominios establecidos (agua, membrana, salmuera). Los modelos utilizados y las simulaciones generadas por Comsol $^{\circ}$ y Matlab fueron adecuadas fenomenológicamente y estadísticamente significativas $(p<$ 0,05 ) en predecir el comportamiento del flux obteniéndose correlaciones mayores al 96\% entre los datos experimentales y los calculados.

\section{FINANCIAMIENTO}

Colciencias. Departamento Administrativo de Ciencia, Tecnología e Innovación.

\section{NOMENCLATURA}

$N \quad$ Flux vapor, masa $\left(\mathrm{kg} \mathrm{m}^{-2} \mathrm{~h}^{-1}\right),\left(\mathrm{mol} \mathrm{m}^{-2} \mathrm{~s}^{-1}\right)$.

$P \quad$ Presión $(\mathrm{Pa})$

$P^{*} \quad$ Presión vapor saturado $(\mathrm{Pa})$

$P_{A l m}$ Presión aire media logarítmica

$Q \quad$ Flux de calor $\left(\mathrm{W} \mathrm{m}^{-2}\right)$

$R \quad$ Constante de los gases $\left(8.314 \mathrm{~J} \mathrm{~K}^{-1} \mathrm{~mol}^{-1}\right)$

$r \quad$ Radio de poro $(\mathrm{m})$

$T$ Temperatura $\left({ }^{\circ} \mathrm{C}, \mathrm{K}\right)$

$x \quad$ Fracción masa $(\mathrm{p} / \mathrm{p} \%)$

\section{Símbolos}

$\varepsilon \quad$ Porosidad

$\delta \quad$ Espesor $(\mathrm{m})$

$\Delta \quad$ Diferencia

$\gamma \quad$ Coeficiente de actividad

$\lambda \quad$ Trayectoria media libre $(\mathrm{m})$

$\mu \quad$ Viscosidad dinámica (Pa s)

$\chi \quad$ Tortuosidad

$\infty \quad$ Valor asintótico

$\varnothing \quad$ Densidad empaquetamiento

$\rho \quad$ Densidad $\left(\mathrm{kg} \mathrm{m}^{-3}\right)$

$\sigma \quad$ Diámetro medio de colisión

\section{Números adimensionales}

$\mathrm{Gz}$ Graetz

Kn Knudsen

$\mathrm{Nu}$ Nusselt

Pr Prandtl

Re Reynolds

Sc Schmidt

Sh Sherwood

\section{REFERENCIAS BIBLIOGRÁFICAS}

Alves, V.D. y Coelhoso, I.M. (2004). Effect of membrane characteristics on mass and heat transfer in the osmotic evaporation process. Journal of Membrane Science, 228(2), 159-167. DOI: 10.1016/j. memsci.2003.10.004

Bailey, A.F.G.; Barbe, A.M.; Hogan, P.A.; Johnson, R.A. y Sheng, J. (2012). The effect of ultrafiltration on the subsequent concentration of grape juice by osmotic distillation. Journal of Membrane Science, 164(1-2), 195-204. DOI: 10.1016/s0376-7388(99)00209-4 
Bui, A. V.; Nguyen, H.M. y Joachim, M. (2003). Prediction of water activity of glucose and calcium chloride solutions. Journal of Food Engineering, 57(3), 243-248. DOI: 10.1016/s0260-8774(02)00304-7

Bui, A.V. y Nguyen, M.H. (2004). Prediction of viscosity of glucose and calcium chloride solutions. Journal of Food Engineering, 62(4), 345-349. DOI: 10.1016/s0260-8774(03)00249-8

Cassano, A.; Drioli, E.; Galaverna, G.; Marchelli, R.; Di Silvestro, G. y Cagnasso, P. (2003). Clarification and concentration of citrus and carrot juices by integrated membrane processes. Journal of Food Engineering, 57(2), 153-163. DOI: 10.1016/ s0260-8774(02)00293-5

Celere, M. y Gostoli, C. (2002). The heat and mass transfer phenomena in osmotic membrane distillation. Desalination, 147(1-3), 133-138.

Courel, M.; Dornier, M.; Herry, J.-M.; Rios, G.M. y Reynes, M. (2000). Effect of operating conditions on water transport during the concentration of sucrose solutions by osmotic distillation. Journal of Membrane Science, 170(2), 281-289.

Courel, M.; Tronel-Peyroz, E.; Rios, G.M.; Dornier, M. y Reynes, M. (2001). The problem of membrane characterization for the process of osmotic distillation. Desalination, 140(1), 15-25.

Chen, B.; Gao, Z.; Jin, W. y Zheng, S. (2011). Analytical mass transfer solution of longitudinal laminar flow of Happel's free surface model. International Journal of Heat and Mass Transfer, 54(17-18), 4000-4008. DOI:http://dx.doi.org/10.1016/j. ijheatmasstransfer.2011.04.025

Domingues, R.C.C.; Ramos, A.A.; Cardoso, V.L. y Reis, M.H.M. (2014). Microfiltration of passion fruit juice using hollow fibre membranes and evaluation of fouling mechanisms. Journal of Food Engineering, 121(0), 73-79. DOl:http://dx.doi.org/10.1016/j. jfoodeng.2013.07.037

Gawronski, R. y Wrzesinska, B. (2000). Kinetics of solvent extraction in hollow-fiber contactors. Journal of Membrane Science, 168(1-2), 213-222. DOI: 10.1016/s0376-7388(99)00317-8

Holz, M.; Heil, S.R. y Sacco, A. (2000). Temperature-dependent self-diffusion coefficients of water and six selected molecular liquids for calibration in accurate $1 \mathrm{H}$ NMR PFG measurements. Physical Chemistry Chemical Physics, 2(20), 4740-4742.

Kujawski, W.; Sobolewska, A.; Jarzynka, K.; Güell, C.; Ferrando, M. y Warczok, J. (2013). Application of osmotic membrane distillation process in red grape juice concentration. Journal of Food Engineering, 116(4), 801-808. DOI:http://dx.doi.org/10.1016/j. jfoodeng.2013.01.033

Lipnizki, F. y Field, R.W. (2001). Mass transfer performance for hollow fibre modules with shell-side axial feed flow: using an engineering approach to develop a framework. Journal of Membrane Science, 193(2), 195-208. DOI: 10.1016/ s0376-7388(01)00512-9

Liu, J. y Wei, J. (2014). Knudsen diffusion in channels and networks. Chemical Engineering Science, 111(0), 1-14. DOl:http://dx.doi.org/10.1016/j. ces.2014.01.014

Manawi, Y.M.; Khraisheh, M.A.M.M.; Fard, A.K.; Benyahia, F. y Adham, S. (2014). A predictive model for the assessment of the temperature polarization effect in direct contact membrane distillation desalination of high salinity feed. Desalination, 341(0), 38-49. DOI:http://dx.doi.org/10.1016/j. desal.2014.02.028

Martínez-Díez, L.; Florido-Díaz, F.J. y Vázquez-González, M.I. (2000). Study of Polarization Phenomena in Membrane Distillation of Aqueous Salt Solutions. Separation Science and Technology, 35(10), 1485-1501.

Mendieta R., M.N.; Montaña P., R.E. y Noguera V., L.A. (2012). Diseño de una cámara de ensayos termoeléctricos. Revista Tecnura, 16, 100-110.

Meng, S.; Ye, Y.; Mansouri, J. y Chen, V. (2014). Fouling and crystallisation behaviour of superhydrophobic nano-composite PVDF membranes in direct contact membrane distillation. Journal of Membrane Science, 463(0), 102-112. DOI:http://dx.doi.org/10.1016/j.memsci.2014.03.027

Naim, R.; Khulbe, K.C.; Ismail, A.F. y Matsuura, T. (2013). Characterization of PVDF hollow fiber membrane for $\mathrm{CO} 2$ stripping by atomic force microscopy analysis. Separation and Purification 
Technology, 109(0), 98-106. DOI:http://dx.doi.org/10.1016/j.seppur.2013.02.036

Nii, S.; Jebson, R.S. y Cussler, E.L. (2002). Membrane evaporators. Journal of Membrane Science, 201(12), 149-159.

Puin Á., H.D.; Noguera V., L.A. y Caicedo M., J.H. (2015). Análisis multifísico de un motor de reluctancia conmutada de rotor exterior usando elementos finitos. Revista Tecnura, 19, 151-156.

Qtaishat, M.; Matsuura, T.; Kruczek, B. y Khayet, M. (2008). Heat and mass transfer analysis in direct contact membrane distillation. Desalination, 219(13), 272-292.

Rahbari-Sisakht, M.; Ismail, A.F.; Rana, D. y Matsuura, T. (2013). Carbon dioxide stripping from diethanolamine solution through porous surface modified PVDF hollow fiber membrane contactor. Journal of Membrane Science, 427(0), 270-275. DOI:http:// dx.doi.org/10.1016/j.memsci.2012.09.060

Rezakazemi, M.; Shirazian, S. y Ashrafizadeh, S.N. (2012). Simulation of ammonia removal from industrial wastewater streams by means of a hollow-fiber membrane contactor. Desalination, 285(0), 383-392. DOI:http://dx.doi.org/10.1016/j. desal.2011.10.030

Romero, J. (2003). Analysis of boundary layer and solute transport in osmotic evaporation. AlChE Journal, 49(11), 2783-2792.

Shaw, P.E.; Lebrun, M.; Dornier, M.; Ducamp, M.N.; Courel, M. y Reynes, M. (2001). Evaluation of Concentrated Orange and Passionfruit Juices Prepared by Osmotic Evaporation. Lebensmittel-Wissenschaft und-Technologie, 34(2), 60-65.

Suárez-Iglesias, O.; Medina, I.; Pizarro, C. y Bueno, J. L. (2008). On predicting self-diffusion coefficients in fluids. Fluid Phase Equilibria, 269(1-2), 80-92. DOI:http://dx.doi.org/10.1016/j.fluid.2008.05.004

Thanedgunbaworn, R.; Jiraratananon, R. y Nguyen, M.H. (2007a). Mass and heat transfer analysis in fructose concentration by osmotic distillation process using hollow fibre module. Journal of Food Engineering, 78(1), 126-135. DOI: 10.1016/j. jfoodeng.2005.09.023
Thanedgunbaworn, R.; Jiraratananon, R. y Nguyen, M.H. (2007b). Shell-side mass transfer of hollow fibre modules in osmotic distillation process. Journal of Membrane Science, 290(1-2), 105-113. DOI: 10.1016/j.memsci.2006.12.021

Thanedgunbaworn, R.; Jiraratananon, R. y Nguyen, M. H. (2009). Vapour Transport Mechanism in Osmotic Distillation Process. International Journal of Food Engineering, 5(5), 1-19. DOI:10.2202/1556-3758.1665

Vaillant, F.; Jeanton, E.; Dornier, M.; O'Brien, G.M.; Reynes, M. y Decloux, M. (2012). Concentration of passion fruit juice on an industrial pilot scale using osmotic evaporation. Journal of Food Engineering, 47(3), 195-202.

Valdés, H.; Romero, J.; Saavedra, A.; Plaza, A. y Bubnovich, V. (2013). Concentration of noni juice by means of osmotic distillation. Journal of Membrane Science, 330(1-2), 205-213.

Viguié, J.; Savart, T.; Duru, P.; Rouch, J.C. y Remigy, J.C. (2013). Characterisation of 3D porous macrostructure of hollow fibre membranes using X-ray tomography-Effects of some spinning process conditions. Journal of Membrane Science, 435(0), 11-20. DOI:http://dx.doi.org/10.1016/j. memsci.2013.01.062

Wahab, A. y Mahiuddin, S. (2001). Isentropic Compressibility and Viscosity of Aqueous and Methanolic Calcium Chloride Solutions. Journal of Chemical \& Engineering Data, 46(6), 1457-1463. DOI:10.1021/ je010072l

Wang, X.-D.; Wang, Z.-X.; Duan, Y.-Y.; An, B. y Lee, D.J. (2014). Efficient evaluation of thermodynamic properties of water and steam on $\mathrm{p}-\mathrm{h}$ surface. Journal of the Taiwan Institute of Chemical Engineers, 45(2), 372-379. DOI:http://dx.doi.org/10.1016/j. jtice.2013.06.016

Wu, J. y Chen, V. (2000). Shell-side mass transfer performance of randomly packed hollow fiber modules. Journal of Membrane Science, 172(1-2), 59-74. DOI: 10.1016/s0376-7388(00)00318-5

Yang, J.; Yu, X.; Yan, J.; Tu, S.-T. y Dahlquist, E. (2013). Effects of $\mathrm{SO} 2$ on $\mathrm{CO} 2$ capture using a hollow fiber membrane contactor. Applied Energy, 
112(0), 755-764. DOI:http://dx.doi.org/10.1016/j. apenergy.2012.11.052

Zhang, L.-Z.; Huang, S.-M.; Chi, J.-H. y Pei, L.-X. (2012). Conjugate heat and mass transfer in a hollow fiber membrane module for liquid desiccant air dehumidification: A free surface model approach. International Journal of Heat and Mass
Transfer, 55(13-14), 3789-3799. DOI:http://dx.doi. org/10.1016/j.ijheatmasstransfer.2012.03.034

Zhao, S.; Feron, P.H.M.; Xie, Z.; Zhang, J. y Hoang, M. (2014). Condensation studies in membrane evaporation and sweeping gas membrane distillation. Journal of Membrane Science, 462(0), 9-16. DOI:http://dx.doi.org/10.1016/j.memsci.2014.03.028

\section{(c) (1) $(\Theta \Theta$

